NASA/TM-2002-211703

Real-Time In Situ Signal-To-Noise Ratio

Estimation for the Assessment of Operational Communications Links

Robert M. Manning

Glenn Research Center, Cleveland, Ohio 
The NASA STI Program Office ... in Profile

Since its founding, NASA has been dedicated to the advancement of aeronautics and space science. The NASA Scientific and Technical Information (STI) Program Office plays a key part in helping NASA maintain this important role.

The NASA STI Program Office is operated by Langley Research Center, the Lead Center for NASA's scientific and technical information. The NASA STI Program Office provides access to the NASA STI Database, the largest collection of aeronautical and space science STI in the world. The Program Office is also NASA's institutional mechanism for disseminating the results of its research and development activities. These results are published by NASA in the NASA STI Report Series, which includes the following report types:

- TECHNICAL PUBLICATION. Reports of completed research or a major significant phase of research that present the results of NASA programs and include extensive data or theoretical analysis. Includes compilations of significant scientific and technical data and information deemed to be of continuing reference value. NASA's counterpart of peerreviewed formal professional papers but has less stringent limitations on manuscript length and extent of graphic presentations.

- TECHNICAL MEMORANDUM. Scientific and technical findings that are preliminary or of specialized interest, e.g., quick release reports, working papers, and bibliographies that contain minimal annotation. Does not contain extensive analysis.

- CONTRACTOR REPORT. Scientific and technical findings by NASA-sponsored contractors and grantees.
- CONFERENCE PUBLICATION. Collected papers from scientific and technical conferences, symposia, seminars, or other meetings sponsored or cosponsored by NASA.

- SPECIAL PUBLICATION. Scientific, technical, or historical information from NASA programs, projects, and missions, often concerned with subjects having substantial public interest.

- TECHNICAL TRANSLATION. Englishlanguage translations of foreign scientific and technical material pertinent to NASA's mission.

Specialized services that complement the STI Program Office's diverse offerings include creating custom thesauri, building customized data bases, organizing and publishing research results ... even providing videos.

For more information about the NASA STI Program Office, see the following:

- Access the NASA STI Program Home Page at http://www.sti.nasa.gov

- E-mail your question via the Internet to help@sti.nasa.gov

- Fax your question to the NASA Access Help Desk at 301-621-0134

- Telephone the NASA Access Help Desk at 301-621-0390

- Write to: NASA Access Help Desk NASA Center for AeroSpace Information 7121 Standard Drive Hanover, MD 21076 
NASA/TM-2002-211703

Real-Time In Situ Signal-To-Noise Ratio

Estimation for the Assessment of

Operational Communications Links

Robert M. Manning

Glenn Research Center, Cleveland, Ohio

National Aeronautics and

Space Administration

Glenn Research Center 
Available from

NASA Center for Aerospace Information 7121 Standard Drive

Hanover, MD 21076
National Technical Information Service 5285 Port Royal Road Springfield, VA 22100

Available electronically at htro//ghtrs grchasa-gov/CLTRS 


\title{
REAL-TIME in situ SIGNAL-TO-NOISE RATIO ESTIMATION FOR THE ASSESSMENT OF OPERATIONAL COMMUNICATIONS LINKS
}

\author{
Robert M. Manning \\ National Aeronautics and Space Administration \\ Glenn Research Center \\ Cleveland, Ohio 44135
}

\section{INTRODUCTION}

The assessment of the propagation conditions that prevail on a communications link, whether it is a point-to-point terrestrial link or an earth-space link, is of vital importance for the optimal operation of the link. The significant indicator of the communications quality of the link is, of course, the signal-to-noise ratio (SNR) $\gamma$. If the value of this quantity goes below a certain given threshold due, for example, to atmospheric conditions such as rain, the bit error rate on the link becomes unacceptable for reliable communications integrity. Knowledge of the dynamic behavior of $\gamma$ is thus essential for the optimal implementation of procedures to mitigate the further degradation of $\gamma$. In many instances, a separate propagation receiver is employed to acquire an associated signal transmitted by a beacon on the communications satellite. Measurement of the fading conditions of this signal level is then extrapolated to that of the communications link. In an effort to ease the operational and financial burden to terminal operation, methods (to be briefly discussed below) have been advanced to estimate $\gamma$ of the communications link by measuring various quantities of the operational communications link itself.

Many methods have been advanced to estimate the SNR using an active modulated communications channels. For example, the output of the receiver matched filter can be sampled, i.e., the voltage level $V_{S}$ for an output symbol, and the value is compared to a pair of a priori determined voltage levels $\pm \alpha, \alpha>0 \quad \alpha<\sqrt{V_{s}}$. The statistical frequency of occurrence $n_{F}$ of values which fail to fall within the interval $[-\alpha, \alpha]$ is calculated as well as the total number $n_{T}$ of samples considered. The ratio $n_{F} / n_{T}$ is related to the symbol error probability by

$$
\frac{n_{F}}{n_{T}}=2 Q\left(\sqrt{k \frac{2 E_{S}}{N_{0}}}\right), \quad k \equiv\left[1-\frac{\alpha}{\sqrt{V_{S}}}\right]
$$

where $E_{S}$ is the symbol energy (i.e., energy per symbol), $N_{0}$ twice the noise power at the output of the matched filter and $Q(\cdots)$ is the well-known $Q$-function. This relationship is then solved for the SNR $\gamma=E_{S} / N_{0}$ by using the inverse function $Q^{-1}(\cdots)$. Although this method has several shortcomings, e.g., limited dynamic range and sensitivity to automatic gain control variations and inter-symbol interference, it suffers from an irreconcilable defect; the inversion of the function $Q(\cdots)$, necessary to obtain the estimate $\hat{\gamma}$ of $\gamma$, is mathematically correct only if $d Q / d \gamma \neq 0$. In the event that $d Q / d \gamma \rightarrow 0$, the problem of determining $\gamma$ becomes ill-posed ${ }^{1}$. That is, a small error in the estimate of $n_{F} / n_{T}$ leads to a large error in the estimate $\hat{\gamma}$. In fact, the ill-posedness of this problem is the major source of the lack of dynamic range. 
An entirely different approach can be imagined which avoids the ill-posedness of that above and thus tends to be more robust in the presence of measurement errors inherent in the sampling of the matched filter output. In the BPSK case, the sampled voltage $V_{S}$ is related to the bi-phase signal amplitude $\pm A$ and the corresponding in-phase noise component $N_{c}$ by

$$
V_{S}=K\left( \pm A+N_{c}\right)
$$

where $K$ is a constant proportionality coefficient incorporating receiver gain factors, etc. Remembering that the goal here is to obtain an expression for the SNR $\gamma=E_{S} / N_{0}$ $=A^{2} / 2 \sigma_{N}^{2}$ where $\sigma_{N}^{2}=\left\langle N_{c}^{2}\right\rangle$ for zero mean, white Gaussian noise, the method endeavors to obtain this ratio solely from the measured values of $V_{S}$. Thus, to separate the noise term, one would try to form the average $\left\langle V_{s}\right\rangle$ using the fact that $\left\langle N_{c}\right\rangle=0$. However, the random bipolar nature of the signal amplitude $A$ also yields a zero average giving $\left\langle V_{S}\right\rangle=0$. The technique that is then adopted in this approach is to form the absolute value $\left|V_{S}\right|$ of each sample and then forming the ensemble average giving

$$
\begin{aligned}
\left\langle\left|V_{S}\right|\right\rangle & =\left\langle\left|K\left( \pm A+N_{c}\right)\right|\right\rangle \\
& \approx K A
\end{aligned}
$$

so long as the condition $A \gg \sigma_{N}$ prevails. Additionally, the sampled values $V_{S}$ are used to compute the variance

$$
\left\langle V_{S}^{2}\right\rangle=K^{2}\left(A^{2}+\sigma_{N}^{2}\right)
$$

Hence, using the former expression to rid to the $A^{2}$ term to give

therefore allowing one to write

$$
\sigma_{N}^{2}=\left\langle V_{S}^{2}\right\rangle-\left\langle\mid V_{S}\right\rangle^{2}
$$

$$
\gamma=\frac{A^{2}}{2 \sigma_{N}^{2}}=\frac{\left\langle\mid V_{S}\right\rangle^{2}}{2\left(\left\langle V_{S}^{2}\right\rangle-\left\langle\mid V_{S}\right\rangle^{2}\right)}
$$

solely in terms of the voltage samples $V_{S}$.

The major drawback of this method is the formation of the absolute value of the random quantity $V_{S}$; such an operation can drastically change the statistical characteristics of the random variable. Even though this was done to rid of the bipolar nature of the communications signal amplitude, it is mathematically faulty and, moreover, needless. One can, and in fact should, incorporate the bipolar nature of the signal amplitude into a rigorous statistical analysis; this characteristic of the signal is just as important as its other aspects. In addition, this approach requires one to use a predetermined bit-stream format within the communications data composed of a series of 1's thus necessitating a synchronization with, for example, a preamble within the modulation format. This results in a further complication of its implementation and, as mentioned above, is unnecessary.

It is the purpose of this work to formulate the rigorous statistical basis for the correct estimation of BPSK signal SNR from what is known about its behavior at the input and output of the receiver demodulator. Instead of employing tacit and unwarranted assumptions concerning the nature of the communication signal for analytical simplification, a complete consistent statistical description of a BPSK signal will be provided to which the well-known techniques of maximum likelihood estimation theory can be applied. By employing, rather than neglecting, all the subtitles of the 
statistics describing the BPSK signal, an unbiased estimation procedure will be derived that makes simple use of its inherent phase characteristics at the demodulator. In what is to follow, a preliminary review of BPSK signal representation will be given which will lay the foundation for the statistical connection between Gaussian noise and SNR. Once an appropriate probabilistic description is obtained that establishes a rigorous contact between SNR and the measured phase error of the BPSK signal entering the receiver demodulator, the methods of maximum likelihood estimation theory will be used to obtain analytical expressions for biased and unbiased estimates of SNR from easily measured phase errors. Finally, the straightforward modifications needed at the demodulator to implement the required phase measurements will be given. It should be noted that the resulting SNR estimation technique is also applicable for a QPSK demodulator simply by applying it to one of the BPSK arms with appropriate modifications for the SNR expression.

\section{PRELIMINARIES OF SIGNAL REPRESENTATION}

Consider the simple BPSK demodulator shown in Figure 1. The phase-modulated signal $s_{i}(t)$ at the input is defined by

$$
s_{i}(t)=A(t) \cos \left(\omega_{0} t+\theta_{i}(t)\right), \quad \theta_{i}(t) \equiv \frac{2 \pi(i-1)}{M}, i=1,2 ; M=2
$$

where $\omega_{0}$ is the angular frequency of the carrier wave, $A(t)$ is its time varying amplitude and $\theta_{i}(t)$ is its bi-phase state. In terms of quadratures, the noise-free BPSK signal is given by

$$
\begin{gathered}
s_{i}(t)=A(t)\left[\cos \left(\omega_{0} t\right) \cos \left(\theta_{i}(t)\right)-\sin \left(\omega_{0} t\right) \sin \left(\theta_{i}(t)\right)\right] \\
=A(t) \cos \left(\omega_{0} t\right) \cos \left(\theta_{i}(t)\right) \\
=A_{c}(t) \cos \left(\omega_{0} t\right), \quad A_{c}(t) \equiv A(t) \cos \left(\theta_{i}(t)\right)
\end{gathered}
$$

where

$$
A_{c}(t) \equiv A(t) \cos \left(\theta_{i}(t)\right)= \pm A(t)
$$

showing the obvious fact that the noise free signal only has an in-phase component $A_{c}(t)$ since the bi-phase states are $\theta_{1}(t)=0^{\circ}, \theta_{2}(t)=180^{\circ}$ thus relegating the sine factors to zero.

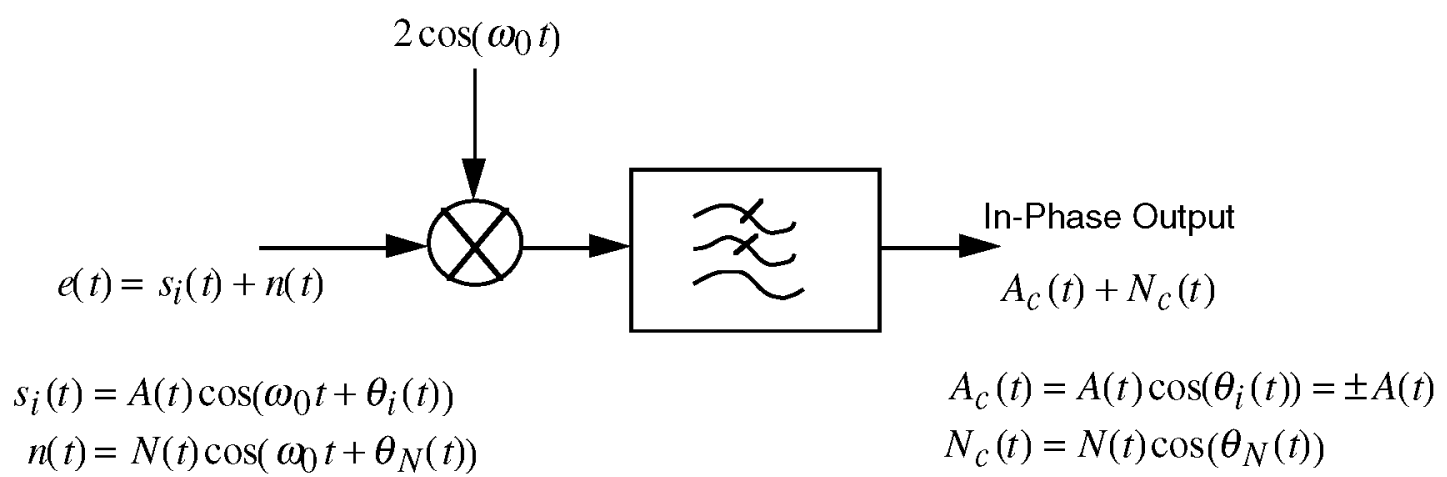

FIGURE 1.

BPSK demodulator showing relationship between signal parameters 
Similarly, the noise $n(t)$ associated with the input is given by

$$
n(t)=N(t) \cos \left(\omega_{0} t+\theta_{N}(t)\right)
$$

where $N(t)$ is the time varying amplitude of the noise and $\theta_{N}(t)$ is the associated phase. In terms of quadratures, the noise is given by

where

$$
\begin{aligned}
n(t) & =N(t)\left[\cos \left(\omega_{0} t\right) \cos \left(\theta_{N}(t)\right)-\sin \left(\omega_{0} t\right) \sin \left(\theta_{N}(t)\right)\right] \\
& =N_{c}(t) \cos \left(\omega_{0} t\right)-N_{s}(t) \sin \left(\omega_{0} t\right)
\end{aligned}
$$

and

$$
N_{c}(t) \equiv N(t) \cos \left(\theta_{N}(t)\right)
$$

$$
N_{s}(t) \equiv N(t) \sin \left(\theta_{N}(t)\right)
$$

showing that, unlike the noise-free BPSK signal component, the noise is characterized by both in-phase and quadrature terms. The composite signal $e(t)$ at the input of the demodulator is given by

$$
\begin{aligned}
e(t)= & s_{i}(t)+n(t) \\
& =\left[A_{c}(t)+N_{c}(t)\right] \cos \left(\omega_{0} t\right)+N_{s}(t) \sin \left(\omega_{0} t\right)
\end{aligned}
$$

where use has been made of Eqs.(2) and (5). Writing the composite signal $e(t)$ in terms of its in-phase and quadrature components and employing Eq.(8) yields

$$
e(t) \equiv E_{c}(t) \cos \left(\omega_{0} t\right)-E_{s} \sin \left(\omega_{0} t\right)
$$

where

$$
E_{c}(t) \equiv A_{c}(t)+N_{c}(t)
$$

and

$$
E_{s}(t) \equiv N_{s}(t)
$$

Writing Eq.(9) in the standard form, in which the signal and noise components are written in Eqs.(1) and (4), finally gives

$$
e(t)=E \cos \left(\omega_{0} t+\theta_{E}(t)\right)
$$

where

$$
E(t)=\sqrt{E_{c}^{2}(t)+E_{s}^{2}(t)}, \quad \theta_{E}(t)=\tan ^{-1}\left[\frac{E_{s}}{E_{c}}\right]
$$

Thus,

$$
E_{c}(t)=E(t) \cos \left(\theta_{E}(t)\right)
$$

and

$$
E_{s}(t)=E(t) \sin \left(\theta_{E}(t)\right)
$$

The geometrical depiction of the various quadrature components dealt with here in the case of the bi-phase signal $s_{1}(t)=A_{c}(t)=A(t) \cos \left(\theta_{1}(t)\right)=+A(t)$ is shown in Figure 2. In this figure, it is easily seen how the addition of the noise $n(t)$ to the signal $s_{1}(t)$ yields a total composite signal $e(t)$ with a phase of $\theta_{E}(t)$ with respect to the in-phase axis. The phase $\theta_{E}(t)$ becomes, in this bi-phase signaling case, the phase error of the received signal $s_{1}(t)$, which represents the input to the demodulator.

Given the preceding development of the relationships of the various signal components that enter into the demodulation of a BPSK signal, the problem to be addressed here is the determination of the associated signal-to-noise ratio 


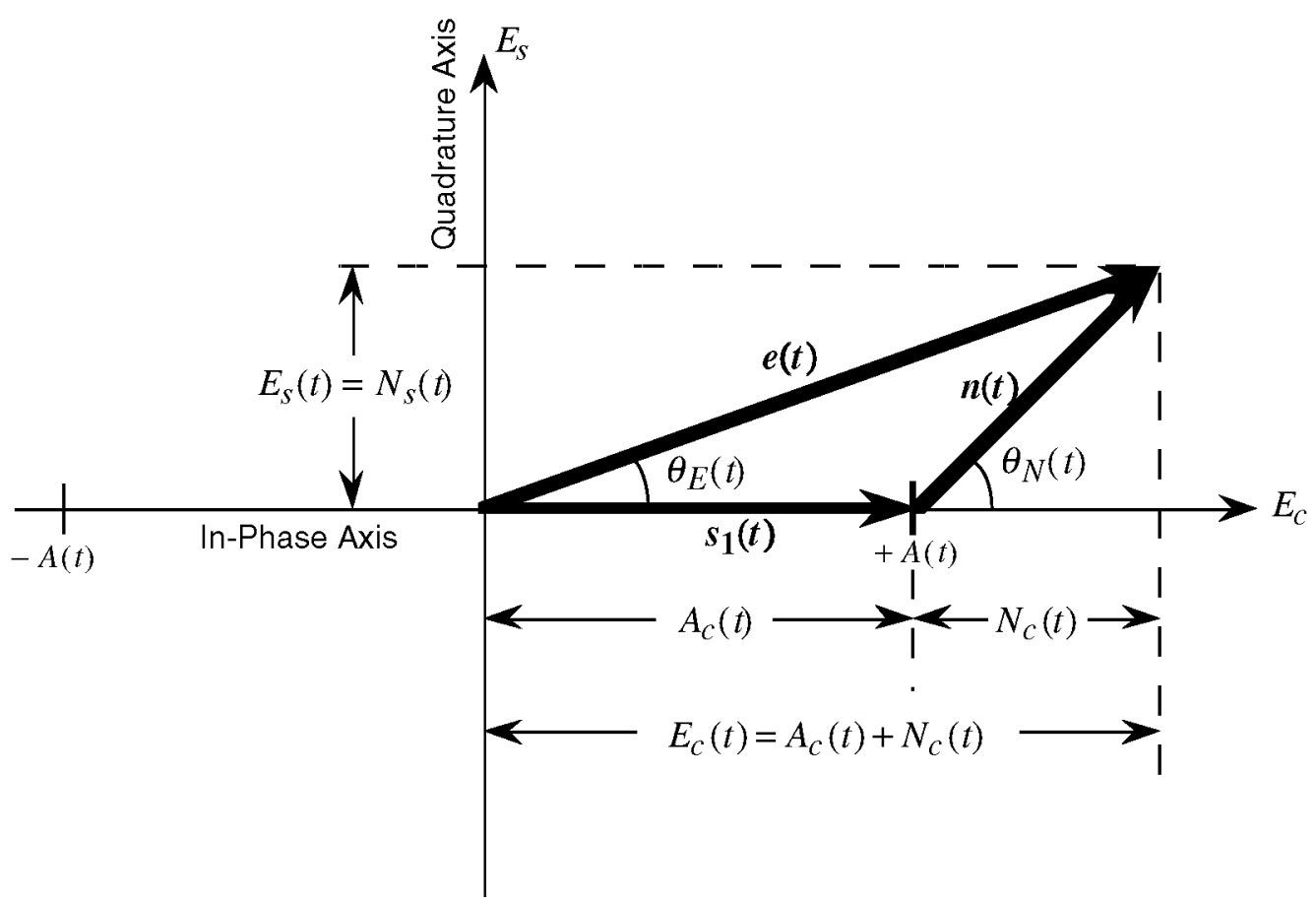

FIGURE 2.

Relationship of the various signal and noise quadrature components of the input of the BPSK demodulator

$$
\gamma(t)=\frac{A^{2}(t)}{\sigma_{N}^{2}(t)}
$$

where the noise variance $\sigma_{N}^{2}$ is given by

$$
\sigma_{N}^{2}(t)=\frac{\left\langle N_{c}^{2}\right\rangle}{2}+\frac{\left\langle N_{s}^{2}\right\rangle}{2}
$$

As shown in the next two sections, this problem can be placed on a mathematically tractable basis and its correct solution rests on the statistical connection between the easily measurable parameters, not of the demodulator output, but of the demodulator input, i.e., $E(t)$ and/or $\theta_{N}(t)$, and the signal-to-noise ratio $\gamma$. The fact that the demodulator input is significant is that it contains both the in-phase and quadrature components of the noise. Additionally, the bi-phase nature of the signal modulation, i.e., $\pm A(t)$, is a major impediment in the application of the methods mentioned above. This aspect of the signal must be properly accounted for in a legitimate treatment of the problem. Such a program begins with the use of what is known about the statistics of the noise process. 


\section{NOISE STATISTICS AND THE SIGNAL-TO-NOISE RATIO}

The noise components $N_{c}(t)$ and $N_{s}(t)$ are random quantities whose statistics are usually taken to be governed by a Gaussian random process. Additionally, it is known that such quadrature components are statistically independent. Letting $\sigma_{N}^{2}(t)$ be the variance of each of these noise components, one has for their joint probability density

$$
p\left(N_{c}(t), N_{s}(t)\right)=\left(\frac{1}{2 \pi \sigma_{N}^{2}(t)}\right) \exp \left[-\frac{N_{c}^{2}(t)+N_{s}^{2}(t)}{2 \sigma_{N}^{2}(t)}\right]
$$

Using Eqs.(10),(11), (14) and (15), one has in terms of the composite signal parameters $E(t)$ and $\theta_{E}(t)$

and

$$
N_{c}(t)=E(t) \cos \left(\theta_{E}(t)\right)-A_{c}(t)
$$

$$
N_{s}(t)=E(t) \sin \left(\theta_{E}(t)\right)
$$

Transforming the probability density function of $N_{c}$ and $N_{s}$ (dropping the time dependence for notational clarity) given by Eq.(16) into one that is a function of $E$ and $\theta_{E}(t)$ involves the transformation

which requires the Jacobian

$$
p\left(E, \theta_{E}\right)=p\left(N_{c}, N_{s}\right)\left|\frac{\partial\left(N_{c}, N_{s}\right)}{\partial\left(E, \theta_{E}\right)}\right|
$$

$$
\left|\frac{\partial\left(N_{c}, N_{s}\right)}{\partial\left(E, \theta_{E}\right)}\right| \equiv\left|\begin{array}{ll}
\frac{\partial N_{c}}{\partial E} & \frac{\partial N_{c}}{\partial \theta_{E}} \\
\frac{\partial N_{s}}{\partial E} & \frac{\partial N_{s}}{\partial \theta_{E}}
\end{array}\right|=E
$$

where Eqs.(17) and (18) where used to obtain the indicated result. Thus, using Eqs.(16)(20), one has in terms of the composite signal parameters

$$
\begin{aligned}
p\left(E, \theta_{E}\right) & =\left(\frac{E}{2 \pi \sigma_{N}^{2}}\right) \exp \left[-\frac{\left(E \cos \theta_{E}-A_{c}\right)^{2}+E^{2} \sin ^{2} \theta_{E}}{2 \sigma_{N}^{2}}\right] \\
& =\left(\frac{E}{2 \pi \sigma_{N}^{2}}\right) \exp \left(-\frac{A_{c}^{2}}{2 \sigma_{N}^{2}}\right) \exp \left[-\frac{E^{2}-2 A_{c} E \cos \theta_{E}}{2 \sigma_{N}^{2}}\right]
\end{aligned}
$$

The signal-to-noise ratio $\gamma$ characterizing the composite signal input to the demodulator is given for the BPSK case as $\gamma(t)=A(t) / \sigma_{N}^{2}(t)$. Writing Eq. (21) in terms of $\gamma$ and remembering Eq.(3) gives

$$
\begin{aligned}
& p\left(E, \theta_{E} \mid \gamma, \theta_{i}, \sigma_{N}\right)=\left(\frac{E}{2 \pi \sigma_{N}^{2}}\right) \exp \left(-\frac{\gamma}{2} \cos ^{2} \theta_{i}\right) . \\
& \cdot \exp \left[-\frac{E^{2}-2 E \sigma_{N} \sqrt{\gamma} \cos \theta_{i} \cos \theta_{E}}{2 \sigma_{N}^{2}}\right]
\end{aligned}
$$

where the density function $p\left(E, \theta_{E}\right)$ is now written as a conditional probability density $p\left(E, \theta_{E} \mid \gamma, \theta_{i}, \sigma_{N}\right)$ governing the values of $E$ and $\theta_{E}$ conditioned on the values of $\gamma, \theta_{i}$, and $\sigma_{N}$. This is done with the hope of being able to obtain an expression, using Eq.(22), connecting the measurable values of $E$ and/or $\theta_{E}$ at the output of the demodulator only 
to the associated value for $\gamma$ thus allowing one to statistically estimate $\gamma$ from such easily measured values.

Having secured the above relationship governing the probability density of the envelope and phase of the composite signal, i.e., $E$ and $\theta_{E}$, and the prevailing values of the signal-to-noise ratio $\gamma$, the variance of the noise power $\sigma_{N}^{2}$, and the phase state of the transmitted signal $\theta_{i}$, one can obtain two relationships involving measurable statistics of $E$, or $\theta_{E}$, and $\gamma$. For example, one can obtain the statistics, conditioned on the value for $\gamma$, that describe the composite signal envelope over all possible signal phase states simply by summing Eq.(22) over the two possible values for $\theta_{i}(t)$ and integrating over all possible values of the phase $\theta_{E}$, viz ${ }^{2}$,

$$
\begin{gathered}
\int_{-\pi}^{\pi} \sum_{i=1}^{2} p\left(E, \theta_{E} \mid \gamma, \theta_{i}, \sigma_{N}\right) d \theta_{E}= \\
=\left(\frac{E}{2 \pi \sigma_{N}^{2}}\right) \exp \left(-\frac{\gamma}{2}\right) \exp \left(-\frac{E^{2}}{2 \sigma_{N}^{2}}\right) \\
\quad \cdot \int_{-\pi}^{\pi}\left\{\exp \left[+\frac{\sqrt{\gamma} E}{\sigma_{N}} \cos \theta_{E}\right]+\exp \left[-\frac{\sqrt{\gamma} E}{\sigma_{N}} \cos \theta_{E}\right]\right\} d \theta_{E} \\
=\left(\frac{E}{\pi \sigma_{N}^{2}}\right) \exp \left(-\frac{\gamma}{2}\right) \exp \left(-\frac{E^{2}}{2 \sigma_{N}^{2}}\right) \int_{0}^{\pi} 2 \cosh \left(\frac{\sqrt{\gamma} E}{\sigma_{N}} \cos \theta_{E}\right) d \theta_{E} \\
=\left(\frac{2 E}{\sigma_{N}^{2}}\right) \exp \left(-\frac{\gamma}{2}\right) \exp \left(-\frac{E^{2}}{2 \sigma_{N}^{2}}\right) I_{0}\left(\frac{\sqrt{\gamma} E}{\sigma_{N}}\right) \\
=p\left(E \mid \gamma, \sigma_{N}\right)
\end{gathered}
$$

which is a form of the well-known generalized Rayleigh distribution for the bi-phase signal envelope. Although this expression is useful for many purposes, it is not sufficient for the estimation of the quantity $\gamma$ from measurable statistics involving $E(t)$ since, as can be seen from Eq.(23), it explicitly involves the parameter $\sigma_{N}^{2}$. Thus, using this approach, one must know, a priori, the prevailing values of $\sigma_{N}^{2}$. Further consideration shows that this must be the case since the noise $n(t)$ has two degrees of freedom which contributes to the composite signal envelope, i.e., $N(t)$ and $\theta_{N}(t)$, as seen from Figure 2. Integrating over all possible values of the phase characterizing the demodulator output leaves the phase of the noise to be determined by knowledge of $\sigma_{N}$. Thus, if one desires to obtain an estimate of $\gamma$ from measurements of $E$ or some statistic related to $E$, one must also need an a priori estimate of $\sigma_{N}$ thus rendering useless a straightforward estimate of $\gamma$ from easily measurable signal parameters. 
However, if one considers statistics that describe the composite signal phase error $\theta_{E}(t)$ by summing Eq.(22) over the two possible values for $\theta_{i}(t)$ and integrating over all possible values of the signal envelope $E(t)$, one obtains ${ }^{3}$

$$
\begin{aligned}
& \int_{0}^{\infty} \sum_{i=1}^{2} p\left(E, \theta_{E} \mid \gamma, \theta_{i}, \sigma_{N}\right) d E= \\
& =\left(\frac{1}{2 \pi \sigma_{N}^{2}}\right) \exp \left(-\frac{\gamma}{2}\right) \\
& \qquad \cdot \int_{0}^{\infty} E \exp \left(-\frac{E^{2}}{2 \sigma_{N}^{2}}\right)\left\{\exp \left[+\frac{\sqrt{\gamma} E}{\sigma_{N}} \cos \theta_{E}\right]+\exp \left[-\frac{\sqrt{\gamma} E}{\sigma_{N}} \cos \theta_{E}\right]\right\} d E \\
& =\left(\frac{1}{\pi \sigma_{N}^{2}}\right) \exp \left(-\frac{\gamma}{2}\right) \int_{0}^{\infty} E \exp \left(-\frac{E^{2}}{2 \sigma_{N}^{2}}\right) \cosh \left(\frac{\sqrt{\gamma} E}{\sigma_{N}} \cos \theta_{E}\right) d E \\
& =\left(\frac{1}{\pi}\right) \exp \left(-\frac{\gamma}{2}\right) \int_{0}^{\infty} r \exp \left(-\frac{r^{2}}{2}\right) \cosh \left(\sqrt{\gamma} r \cos \theta_{E}\right) d r \\
& =\sqrt{\frac{1}{2 \pi}} \sqrt{\gamma} \cos \left(\theta_{E}\right) \exp \left(-\frac{\gamma}{2} \sin ^{2} \theta_{E}\right) \operatorname{erf}\left(\sqrt{\frac{\gamma}{2}} \cos \theta_{E}\right)+\frac{1}{\pi} \exp \left(-\frac{\gamma}{2}\right) \\
& =p\left(\theta_{E} \mid \gamma\right)
\end{aligned}
$$

where the third integral results from the change of variables $r \equiv E / \sigma_{N}$ and $\operatorname{erf}(\cdots)$ in the fourth line is the 'error function' or probability integral defined by

$$
\operatorname{erf}(x) \equiv \frac{2}{\sqrt{\pi}} \int_{0}^{x} \exp \left(-t^{2}\right) d t
$$

This formulation does not involve any a priori information other than the signal-to-noise ratio $\gamma$. Thus, Eq.(24) gives a relationship involving the conditional probability density of the phase of the demodulator output given a value for the signal-to-noise ratio $\gamma$ of the composite signal, with no other a priori information necessary. This expression is indeed a candidate for the basis of estimating $\gamma$ from knowledge of values for $\theta_{E}$. The proper mathematical foundation for this procedure will now be given.

\section{THE MAXIMUM LIKELIHOOD ESTIMATION OF SIGNAL-TO-NOISE RATIO FROM PHASE MEASUREMENTS OF THE BPSK DEMODULATOR INPUT}

Equation (24), giving the conditional probability density governing the values of $\theta_{E}$ given a prevailing value for $\gamma$, can formally be written in the opposite sense, i.e., a conditional probability density governing the values of $\gamma$ given a prevailing value for $\theta_{E}$, viz.,

$$
p\left(\gamma \mid \theta_{E}\right)=\sqrt{\frac{1}{2 \pi}} \sqrt{\gamma} \cos \left(\theta_{E}\right) \exp \left(-\frac{\gamma}{2} \sin ^{2} \theta_{E}\right) \operatorname{erf}\left(\sqrt{\frac{\gamma}{2}} \cos \theta_{E}\right)+\frac{1}{\pi} \exp \left(-\frac{\gamma}{2}\right)
$$

Although not explicitly shown in Eq.(25) for notational simplicity, all signal components that enter into this expression are still functions of time, $\theta_{E}=\theta_{E}(t)$, etc. Assuming that a time series of statistically independent phase measurements $\theta_{E}\left(t_{1}\right), \theta_{E}\left(t_{2}\right), \cdots, \theta_{E}\left(t_{k}\right)$ can be obtained from the composite signal input to the demodulator that subtend a time interval $\Delta t=t_{k}-t_{1}$ whose value is small enough such that $\gamma(t)$ and $\sigma(t)$ can be taken to 
be constant in $\Delta t$, one can use Eq.(25) to obtain a probability density function conditioned on the series of phase measurements given by

$$
p\left(\gamma \mid \theta_{E}\left(t_{1}\right), \theta_{E}\left(t_{2}\right), \cdots, \theta_{E}\left(t_{k}\right)\right) \equiv \prod_{j=1}^{k} p\left(\gamma \mid \theta_{E}\left(t_{j}\right)\right)
$$

From this, one can form the likelihood functional defined by

$$
\begin{gathered}
L\left(\gamma \mid \theta_{E}\left(t_{1}\right), \theta_{E}\left(t_{2}\right), \cdots, \theta_{E}\left(t_{k}\right)\right) \equiv \ln \left\{p\left(\gamma \mid \theta_{E}\left(t_{1}\right), \theta_{E}\left(t_{2}\right), \cdots, \theta_{E}\left(t_{k}\right)\right)\right\} \\
=\sum_{j=1}^{k} \ln \left\{p\left(\gamma \mid \theta_{E}\left(t_{j}\right)\right)\right\}
\end{gathered}
$$

According to the method of maximum likelihood, the corresponding estimate $\gamma^{*}$ of $\gamma$ is found from this functional as that value of $\gamma$ for which $L(\cdots)$ is a maximum. Hence, the estimate $\gamma^{*}$ of $\gamma$ which prevails over the set of phase measurements $\theta_{E}\left(t_{1}\right), \theta_{E}\left(t_{2}\right)$, $\cdots, \theta_{E}\left(t_{k}\right)$, of a BPSK modulated signal is given by

$$
\left.\frac{\partial L\left(\gamma \mid \theta_{E}\left(t_{1}\right), \theta_{E}\left(t_{2}\right), \cdots, \theta_{E}\left(t_{k}\right)\right)}{\partial \gamma}\right|_{\gamma=\gamma^{*}}=0
$$

It is important to remember that the bipolar nature of the modulated signal as well as the usual assumptions of Gaussian noise are already convolved in the probabilistic description of Eq.(25). Also, unlike the general case where $\gamma$ and $\sigma_{N}^{2}$ are functions of time, they can now be taken as constant and independent of time during sufficiently small measurement intervals $\Delta t$. A quantitative measure for 'sufficiently small' will be given below.

It is now necessary to find the root $\gamma^{*}$ of Eq.(28). Using Eqs.(25)-(27) in Eq.(28), and, for analytical tractability, neglecting the last term of Eq.(25) (an approximation which holds for large values of $\gamma$ ), and performing the required differentiation yields

$$
\begin{aligned}
\left.\frac{\partial L}{\partial \gamma}\right|_{\gamma=\gamma^{*}}=0=\frac{k}{2}\left(\frac{1}{\gamma^{*}}\right)-\frac{1}{2} \sum_{i=1}^{k} & \sin ^{2}\left(\theta_{E}\left(t_{i}\right)\right)+ \\
& +\left.\sum_{i=1}^{k} \frac{\partial}{\partial \gamma}\left\{\ln \left(\operatorname{erf}\left(\sqrt{\frac{\gamma}{2}} \cos \left(\theta_{E}\left(t_{i}\right)\right)\right)\right)\right\}\right|_{\gamma=\gamma^{*}}
\end{aligned}
$$

Completing the differentiation of the last term results in a rather unwieldy expression. Using the fact that the $\operatorname{erf}(\cdots)$ function tends to a constant for large values of the argument, i.e., for large $\gamma$, one can neglect this term (consistent with the neglect of the second term of Eq.(25)) and find that in this case, the optimal estimate for $\gamma$ of a biphase signal with additive Gaussian noise based on a series of $k$ phase measurements $\theta_{E}\left(t_{i}\right)$ of the composite signal input is given by

$$
\gamma^{*}=\left[\frac{1}{k} \sum_{i=1}^{k} \sin ^{2}\left(\theta_{E}\left(t_{i}\right)\right)\right]^{-1}, \quad \gamma>>1
$$

Because of the approximations involved with neglecting the second term of Eq.(25) and the third term of Eq.(29), this maximum likelihood estimate is biased toward large values of $\gamma$. This bias must now be removed for small values of $\gamma$. To this end, one uses the 
original expression of Eq.(25) retaining the second term since this will be appreciable for small $\gamma$. However, to maintain analytical flexibility, the $\operatorname{erf}(\cdots)$ function of Eq.(25) will continued to be neglected. Finally, the analysis becomes more amenable if the inverse function $1 / \gamma^{*}$ given by Eq.(30) is used. Given these considerations, one has ${ }^{4}$ for the unbiased estimate $\hat{\gamma}$ of $\gamma *$ over all possible values of the phase $\theta_{E}(t)$

$$
\begin{aligned}
\left\langle\frac{1}{\gamma^{*}}\right\rangle=\int_{-\pi / 2}^{\pi / 2} \sin ^{2}\left(\theta_{E}\right) p\left(\hat{\gamma} \mid \theta_{E}\right) d \theta_{E} & \\
= & \left(\frac{2}{\pi}\right) \exp \left(-\frac{\hat{\gamma}}{2}\right) \int_{0}^{\pi / 2} \sin ^{2} \theta_{E} d \theta_{E}+ \\
& +2 \sqrt{\frac{1}{2 \pi}} \sqrt{\hat{\gamma}} \int_{0}^{\pi / 2} \sin ^{2}\left(\theta_{E}\right) \cos \left(\theta_{E}\right) \exp \left(-\frac{\hat{\gamma}}{2} \sin ^{2}\left(\theta_{E}\right)\right) d \theta_{E} \\
= & \left(\frac{1}{2}-\sqrt{\frac{2}{\pi}} \sqrt{\frac{1}{\hat{\gamma}}}\right) \exp \left(-\frac{\hat{\gamma}}{2}\right)+\left(\frac{1}{\hat{\gamma}}\right) \operatorname{erf}\left(\sqrt{\frac{\hat{\gamma}}{2}}\right)
\end{aligned}
$$

Thus, the unbiased estimate $\hat{\gamma}$ of $\gamma$ is related to the biased estimate $\gamma^{*}$, given by Eq.(30), through the non-linear relationship of Eq.(31). (It should be mentioned that the ensemble average of $\sin ^{2}\left(\theta_{E}\right)$ is calculated over the phase interval $-\pi / 2 \leq \theta_{E} \leq \pi / 2$ since $p\left(\hat{\gamma} \mid \theta_{E}\right)$ is periodic in $\theta_{E}$ with period $\pi$. (Hence, phase values that differ by $\pm \pi$ essentially correspond to the same SNR values and the averaging interval chosen in Eq.(31) implements the separate identification of these intervals.) Since there are no other parameters of the problem that enter into Eq.(31), this relationship is universal for BPSK modulation and is plotted in Figure 3. From Eq.(31) one has the following limiting behavior:

$$
\lim _{\hat{\gamma} \rightarrow 0}\left\langle\frac{1}{\gamma^{*}}\right\rangle=\frac{1}{2}, \quad \lim _{\hat{\gamma} \rightarrow \infty}\left\langle\frac{1}{\gamma^{*}}\right\rangle=\frac{1}{\hat{\gamma}}
$$

Thus, for large $\hat{\gamma}$, the two estimates converge for the reasons explained above. In the case of small $\hat{\gamma}$, the biased estimate approaches 2 since in this case, the phase errors $\theta_{E}\left(t_{i}\right)$ are randomly distributed within their range $-\pi / 2 \leq \theta_{E}\left(t_{i}\right) \leq \pi / 2$. Note that Eq.(31) does not monotonically approach the value of 0.5 as $\gamma \rightarrow 0$; it overshoots the 0.55 level before it turns toward the 0.5 limit. This defect is due to the approximation made above in the neglect of the $\operatorname{erf}(\cdots)$ function in Eq.(29). A more careful analysis may correct this shortcoming.

The implementation of this process is straightforward. Considering now the composite signal input to the demodulator in terms of Eqs.(9), and (14)-(15), instead of the previous representation of Eq.(8), and keeping in mind the goal of securing the values of the phase error $\theta_{E}(t)$, one arrives at the required modifications shown in Figure 4. As $k$ sampled values of $\theta_{E}(t)$ are obtained (just how to determine a value for $k$ will be discussed below), one then implements Eq.(30) to obtain the biased estimate $\gamma *$ of the signal-to-noise ratio $\gamma$. Once this has been obtained, Eq.(31) is employed and solved for the corresponding unbiased estimate $\bar{\gamma}$; this latter procedure can be effected by use of a 'look-up' table that represents the universal graphical behavior of Figure 3. 


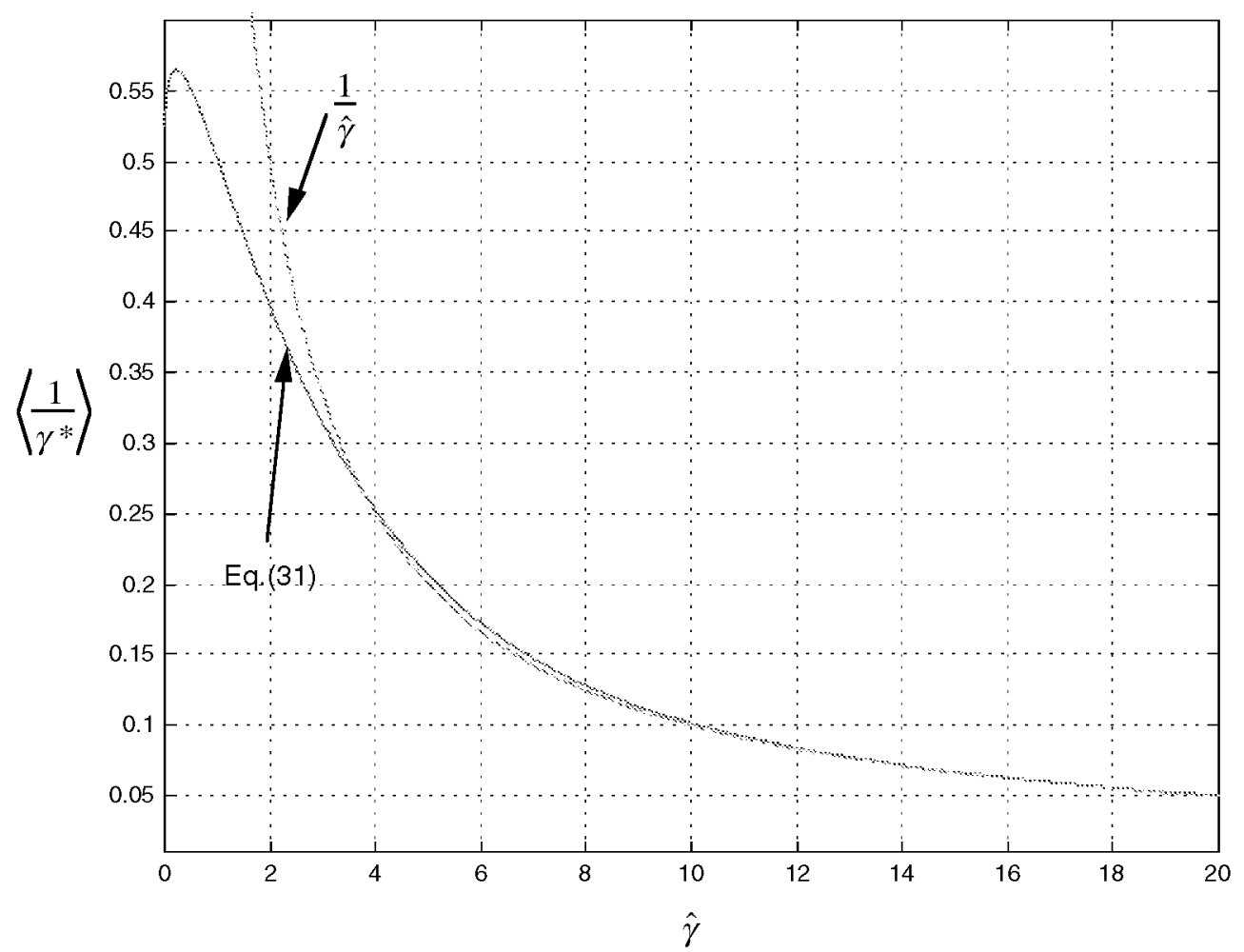

FIGURE 3.

Characteristic curve relating the biased estimate $\gamma^{*}$, calculated from phase error measurements $\theta_{E}\left(t_{i}\right)$ according to Eq.(31), to the unbiased estimate $\hat{\gamma}$.

\section{DETERMINATION OF PHASE SAMPLING LENGTH TO ASSURE CONSTANCY OF $\gamma$ AND $\sigma_{N}^{2}$ DURING THE INTERVAL}

It is imperative that the prevailing values for $\gamma$ and $\sigma_{N}^{2}$ do not evolve during the time period in which the $k$ samples of composite signal phase $\theta_{E}\left(t_{i}\right)$ are formed to obtain the biased estimate $\gamma^{*}$. The fundamental constraint which must be addressed during the measurement process is the fact that $\gamma$ does not have spectral components that exceed 20 $\mathrm{Hz}$ for an atmospheric channel. (This is the upper limit for atmospheric scintillation at communications satellite frequencies.) Thus, if the data rate of the communications link is $R_{D}$ bps, and if a measurement of the phase $\theta_{E}\left(t_{i}\right)$ occurs for $k$ consecutive bit intervals, one must satisfy the Nyquist sampling constraint

$$
\frac{R_{D}}{k} \geq 40 \mathrm{~Hz}
$$

This inequality serves to bound the number of phase samples. 


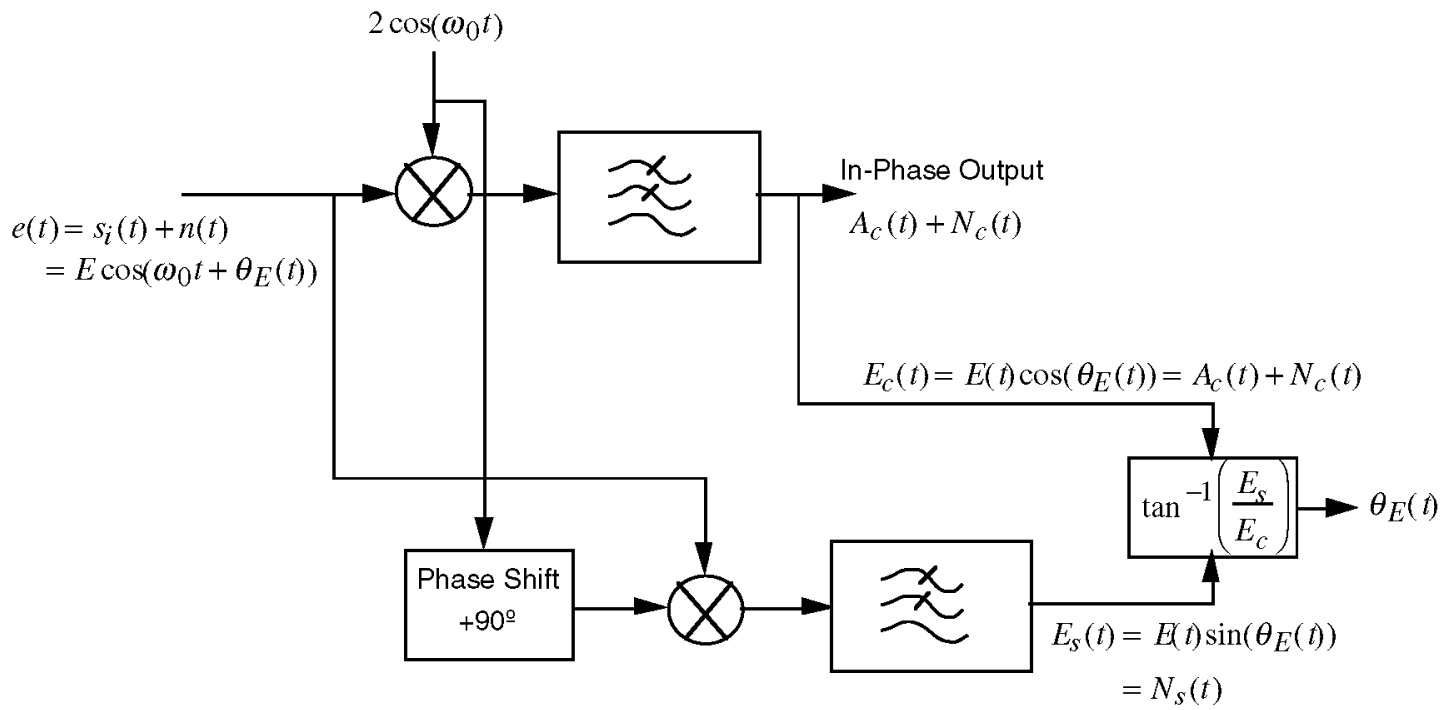

FIGURE 4

Modification to BPSK demodulator to obtain phase error in composite signal.

\section{EXTENSION TO THE GENERAL M-ARY PHASE MODULATION CASE}

The simplest application of the foregoing to QPSK and other M-ary phase modulated cases involves using the method shown in Figure 4 in conjunction with Eq.(30) with just one of the arms of the demodulator. In the interest of maintaining mathematical rigor, one could return to the analysis given above and employ, e.g., $M=4$ in Eq.(1) for the QPSK case. Thus, Eq.(24) would then employ a sum over $i=4$ possible phase states. This, however, would complicate the SNR estimate analogous to that of Eq.(30) for the BPSK case.

\section{REFERENCES}

1. A. N. Tikhonov and V. Y. Arsenin, Solutions of Ill-Posed Problems (John Wiley \& Sons, New York, 1977).

2. I. S. Gradshteyn and I. M. Ryzhik, Table of Integrals, Series, and Products (Academic Press, New York, 1980). Eq.(3.534.2).

3. Ref. 2, Eq.(3.562.4).

4. Ref. 2, Eq.(3.381.1) and use of the simplifying transformations embodied in Eqs.(8.356.1) and (8.359.4). 


\section{\begin{tabular}{l|l|l|} 
1. AGENCY USE ONLY (Leave blank) & 2. REPORT DATE & 3. REPORT TYPE AND DATES COVERED
\end{tabular}}

\begin{tabular}{|c|c|c|}
\hline & August 2002 & Technical Memorandum \\
\hline
\end{tabular}

\section{\begin{tabular}{|l|l} 
4. TITLE AND SUBTITLE & 5. FUNDING NUMBERS
\end{tabular}}

Real-Time In Situ Signal-To-Noise Ratio Estimation for the Assessment of

Operational Communications Links

6. AUTHOR(S)

WU-727-01-10-00

Robert M. Manning

7. PERFORMING ORGANIZATION NAME(S) AND ADDRESS(ES)

National Aeronautics and Space Administration

John H. Glenn Research Center at Lewis Field

Cleveland, Ohio 44135-3191

9. SPONSORING/MONITORING AGENCY NAME(S) AND ADDRESS(ES)

10. SPONSORING/MONITORING

AGENCY REPORT NUMBER

National Aeronautics and Space Administration

Washington, DC 20546-0001

NASA TM-2002-211703

\section{SUPPLEMENTARY NOTES}

Responsible person, Robert M. Manning, organization code 5640, 216-433-6750.

12a. DISTRIBUTION/AVAILABILITY STATEMENT

12b. DISTRIBUTION CODE

Unclassified - Unlimited

Subject Categories: 04, 17, and 32

Distribution: Nonstandard

Available electronically at http:/gltrs.grc.nasa.gov/GLTRS

This publication is available from the NASA Center for AeroSpace Information, 301-621-0390.

13. ABSTRACT (Maximum 200 words)

The work presented here formulates the rigorous statistical basis for the correct estimation of communication link SNR of a BPSK, QPSK, and for that matter, any M-ary phase-modulated digital signal from what is known about its statistical behavior at the output of the receiver demodulator. Many methods to accomplish this have been proposed and implemented in the past but all of them are based on tacit and unwarranted assumptions and are thus defective. However, the basic idea is well founded, i.e., the signal at the output of a communications demodulator has convolved within it the prevailing SNR characteristic of the link. The acquisition of the SNR characteristic is of the utmost importance to a communications system that must remain reliable in adverse propagation conditions. This work provides a correct and consistent mathematical basis for the proper statistical 'deconvolution' of the output of a demodulator to yield a measure of the SNR. The use of such techniques will alleviate the need and expense for a separate propagation link to assess the propagation conditions prevailing on the communications link. Furthermore, they are applicable for every situation involving the digital transmission of data over planetary and space communications links.

\section{SUBJECT TERMS}

Communication theory; Signal fading; Likelihood ratio

\begin{tabular}{|c|c|}
\hline $\begin{array}{c}\text { 17. SECURITY CLASSIFICATION } \\
\text { OF REPORT }\end{array}$ & $\begin{array}{c}\text { 18. SECURITY CLASSIFICATION } \\
\text { OF THIS PAGE } \\
\text { Unclassified }\end{array}$ \\
Unclassified
\end{tabular}

NSN 7540-01-280-5500

\begin{tabular}{|l|l|} 
& $\begin{array}{c}15 . \text { NUMBER OF PAGES } \\
18\end{array}$ \\
\cline { 2 - 3 } & 16. PRICE CODE \\
$\begin{array}{l}\text { 19. SECURITY CLASSIFICATION } \\
\text { OF ABSTRACT } \\
\text { Unclassified }\end{array}$ & 20. LIMITATION OF ABSTRACT \\
\hline \multicolumn{2}{|c|}{$\begin{array}{l}\text { Standard Form 298 (Rev. 2-89) } \\
\text { Prescribed by ANSI Std. Z39-18 } \\
298-102\end{array}$} \\
\hline
\end{tabular}

